\title{
EXTREME STARBURST OR CENTRAL ACTIVITY IN X-RAY LUMINOUS IRAS GALAXIES
}

\author{
Th. BOLLER ${ }^{1}$ and M. DENNEFELD ${ }^{2}$ \\ ${ }^{1}$ Max-Planck Institut für Extraterrestrische Physik, D-85748 Garching bei München, \\ Germany \\ ${ }^{2}$ Institut d'Astrophysique, 98 bis Boulevard Arago, F-75014 Paris, France
}

\begin{abstract}
ROSAT All Sky Survey observations of IRAS galaxies have revealed up to now a number of (10) optically non-Seyfert galaxies with X-ray $(0.1-2.4 \mathrm{keV})$ luminosities up to a few $10^{13} \mathrm{erg} \cdot \mathrm{s}^{-1}$ (Boller et al. 1992). The sources are brighter than previous detection limits of a few $10^{41} \mathrm{erg} \cdot \mathrm{s}^{-1}$ as found by Stocke et al. (1991) or Green, Anderson \& Ward (1992) for Einstein sources. The optical classification is based on follow-up observations which indicate clearly the non-Seyfert (LINER and HII region-like galaxies) nature. Our investigations reveal that galaxies classified as non-Seyferts on the basis of optical spectroscopy may reach exceptionally high X-ray luminosities which are similar to that of Seyfert galaxies. On the basis of the present observational material we suppose a hidden low luminosity AGN in the centre of these objects as the source of energy production. The objects are of interest when evaluating starburst versus central activity.
\end{abstract}

\section{X-Ray Luminous Galaxies}

A number of ROSAT spiral galaxies (10) classified by follow-up spectroscopy as HII or LINER galaxies reach X-ray luminosities up to a few $10^{43} \mathrm{erg} \cdot \mathrm{s}^{-1}$ at least one order of magnitude higher than that detected with Einstein $\left(4 \cdot 10^{41} \mathrm{erg} \cdot \mathrm{s}^{-1}\right.$ [Stocke et al. 1992] or $3 \cdot 10^{41} \mathrm{erg} \cdot \mathrm{s}^{-1}$ [Green et al. 1992]). The object names, the $X$-ray luminosities and the classification from optical followup spectroscopy can be found in Boller et al. (1993).

\section{Starburst versus Central Activity}

We perform model calculation on how much X-ray emission can come from a starburst. We find that the integrated emission of stellar X-ray emitters can not exceed about $9 \cdot 10^{48}$ erg per one solar mass of gas consumed into stars (Fig. 1). This result is compared with observations of normal and Seyfert galaxies, as well as high X-ray luminous $\left(L(0.1-2.4 \mathrm{keV})>10^{42} \mathrm{erg} \cdot \mathrm{s}^{-1}\right)$ HII and LINER galaxies detected in the ROSAT All Sky Survey. The HII and LINER galaxies reach up to a few $10^{50} \mathrm{erg} \cdot M \sigma^{\prime}$. This amount of emitted energy can not be explained with stellar $X$-ray emitters even by changing the model parameters within reasonable limits. It can be explained if we assume a contribution of an active nucleus in addition to the $\mathrm{X}$-ray emission of stellar contributors. 


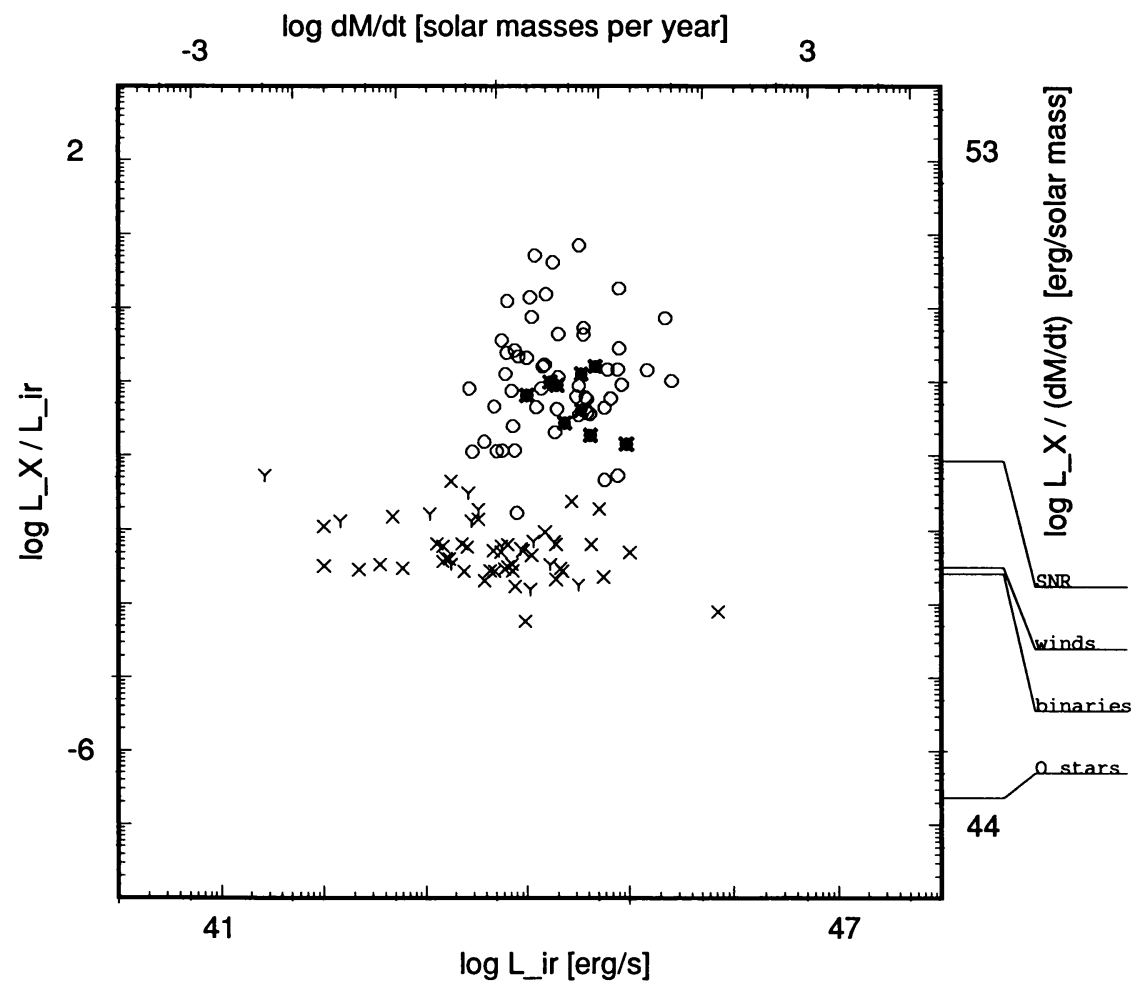

Figure 1. Ratio of X-ray to far-infrared luminosity versus far-infrared luminosity. The X-ray luminous HII and LINER galaxies (fat stars) show a similar distribution of Seyfert galaxies (open circles). The efficiency of producing $\mathrm{X}$-rays per one solar mass of gas consumed into stars is marked for different stellar $\mathrm{X}$-ray contributors.

\section{References}

Boller, T.H., Meurs, EJ.A., Brinkmann, W., Fink, H., Zimmermann and Adorf, H.-M., 1992. Astron. Astrophys., 261, 57.

Boller, T.H., Meurs, E.J.A., Dennefeld, M. and Fink, H., 1993. Astrophys \& Space Science, 205, 43.

David, L.P., Jones, C. and Forman, W., 1992. Astrophys. J., 388, 82.

Green, P.J., Anderson, S.F. and Ward, M.J., 1992. Mon. Not. R. astron. Soc., 254, 30.

Stocke et al. 1991. Astrophys. J., 76, 813. 